\section{Harmonics terminated L-band frequency quadrupler using composite right/ left-handed transmission line}

H. Choi, J. Lim and Y. Jeong

A design method for an L-band frequency quadrupler with composite right/left-handed transmission line is proposed. The left-handed transmission line in the proposed frequency quadrupler suppresses the fundamental component $\left(f_{0}\right)$, while the composite right/left-handed $\lambda / 4$ open stub reduces unwanted higher-order harmonics. From the experimental results, unwanted harmonics are suppressed to achieve harmonic distortion of $51 \mathrm{dBc}$ minimum with output phase noise of $-94.34 \mathrm{dBc} / \mathrm{Hz}$ at $10 \mathrm{kHz}$ offset.

Introduction: High-frequency signal sources can be obtained by multiplying the low-frequency signal that has relatively high stability and low phase noise. In general, frequency multipliers include the unwanted $f_{0}$ and harmonic components $\left(n f_{0}\right)$, and the noise characteristic of the fundamental signal directly influences the multiplied frequency components. [1,2]. The composite right/left-handed transmission line (CRLH TL) that is usually employed as the lumped equivalent circuit for various microwave circuits has been applied to the design of a dual-band passive circuit, in which the phase response of the CRLH TL is manipulated to yield electrical lengths of $\lambda / 4$ and $3 \lambda / 4$ at two arbitrary frequencies [3-6].

In this Letter, we propose a harmonic suppression technique for a frequency quadrupler using a left-handed (LH) and CRLH TL to suppress the unwanted harmonic components. We have implemented and measured the frequency quadrupler, and present the experimental results with discussion.

Frequency quadrupler design using CRLH TL: From the reduced conduction angle analysis, the amplitude of the fourth harmonic is maximised when the conduction angle is around $60^{\circ}$ [7]. Therefore, the bias point for the frequency multiplier should be selected near the pinch-off, between class B and C. Once the bias condition is determined, the input and output ports have to be matched for $f_{0}$ and the desirable higher-order harmonic component $\left(4 f_{0}\right)$, respectively. Fig. 1 shows the proposed frequency quadrupler structure.

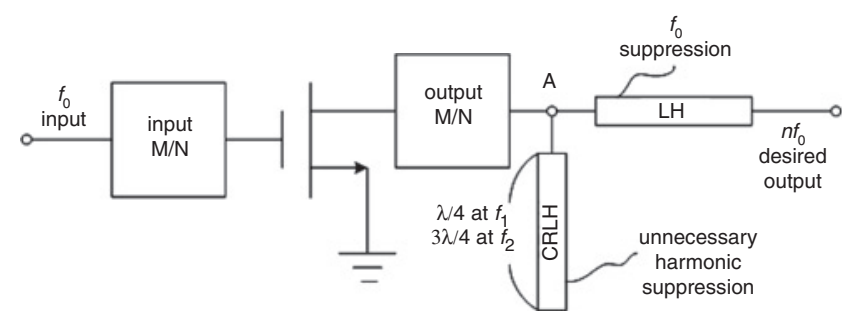

Fig. 1 Block diagram of proposed L-band frequency quadrupler

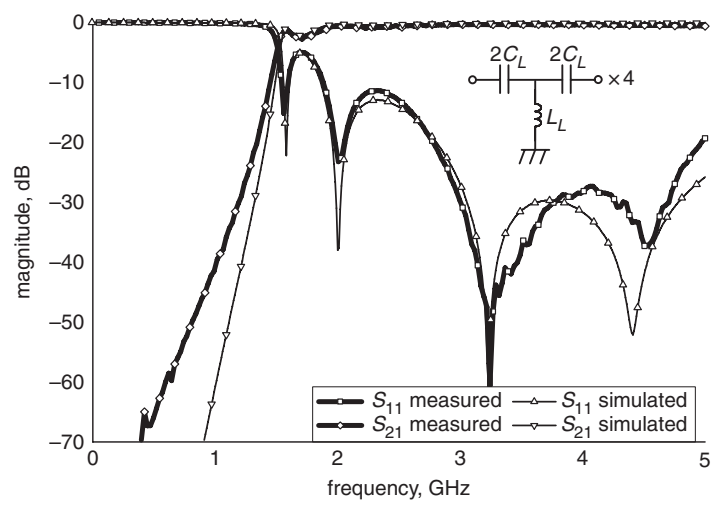

Fig. 2 Simulated and measured result of $L H T L$

The LH TL can be implemented by lumped elements. Since the electrical characteristic of the LH TL is similar to that of highpass filters, it is useful in the suppression of $f_{0}$. The LH TL has been designed to have four unit cells, and the element values are $C_{L}=1.1 \mathrm{pF}$ and $L_{L}=$
$2.8 \mathrm{nH}$. The characteristic impedance of the LH TL is about $50.5 \Omega$. Fig. 2 shows the simulation and measurement results of the designed LH TL, where $f_{0}$ at $1 \mathrm{GHz}$ can be suppressed over $40 \mathrm{~dB}$.

The Phase response of the CRLH TL should be $-90^{\circ}$ at $f_{1}$ and $-270^{\circ}$ at $f_{2}$, where $f_{1}$ and $f_{2}$ are arbitrary frequencies within the range $1<f_{2} / f_{1}<2$. Calculated values of $\mathrm{LH}$ cells $\left(C_{L}\right.$ and $\left.L_{L}\right)$ are obtained by equations in [3], and they are $0.6 \mathrm{pF}$ and $1.7 \mathrm{nH}$ for $N=1$, respectively. Simulated and measured results using these values with the implemented circuit schematic are shown in Fig. 3. Second and third harmonics are suppressed over $24 \mathrm{~dB}$, insertion loss at the fourth harmonic being $0.24 \mathrm{~dB}$.

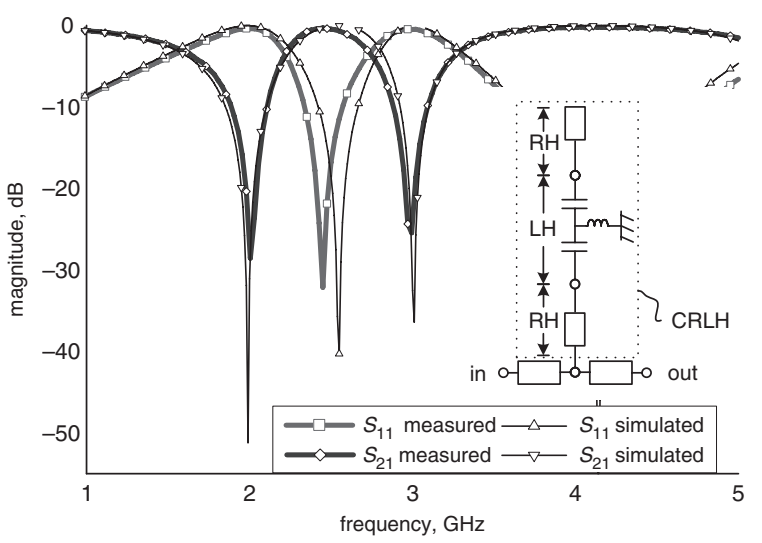

Fig. 3 Simulated and measured results of CRLH $\lambda / 4$ open stub with circuit schematic

Experimental results and discussion: To show the validity, we designed the L-band frequency quadrupler with $f_{0}$ of $1 \mathrm{GHz}$, and the desired output is $4 \mathrm{GHz}$ with harmonics at 2 and $3 \mathrm{GHz}$ being unnecessary components. Therefore, $f_{1}$ and $f_{2}$ of the CRLH $\lambda / 4$ open stub should be 2 and $3 \mathrm{GHz}$ in this case.
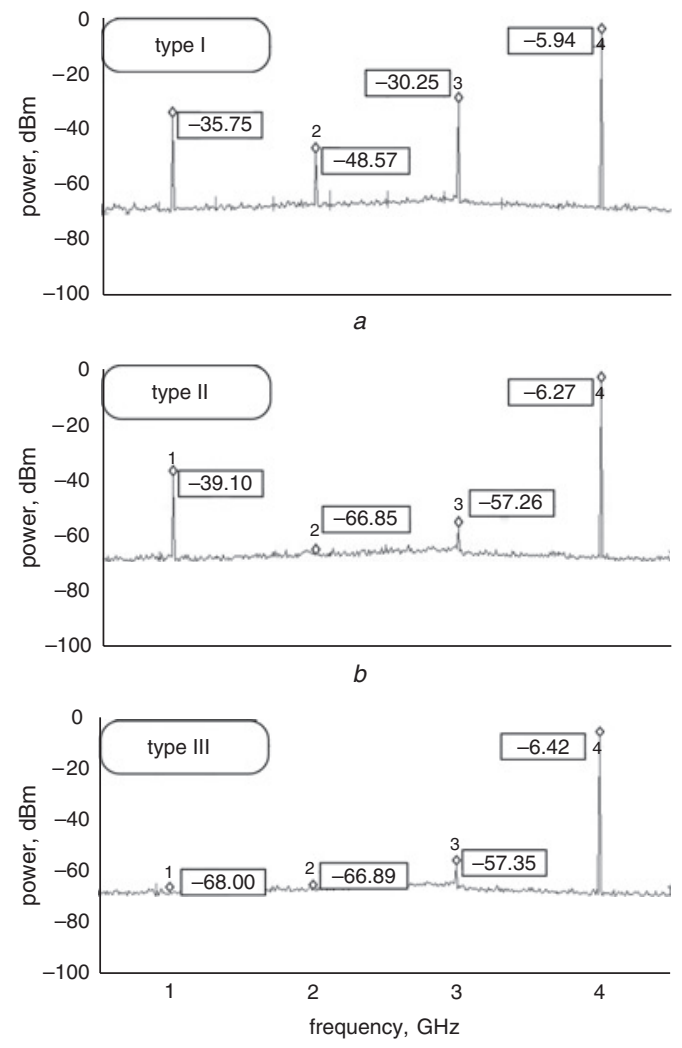

$c$

Fig. 4 Measured output spectrum of frequency quadrupler a Type I network (without any harmonic termination circuit) $b$ Type II network (only with OS)

$c$ Type III network (type II network with LH TL) 
Fig. 4 shows the measured output spectrum of the frequency quadrupler. To analyse the effect of the proposed harmonic termination circuit, we have implemented three different types of frequency quadrupler: a frequency quadrupler without any harmonic termination circuit (only with input/output matching) as type I, a frequency quadrupler only with CRLH $\lambda / 4$ open stub (OS) TL as type II, and a frequency quadrupler with OS and the LH TL (proposed structure) as type III. Signal powers at $f_{0}, 2 f_{0}$, and $3 f_{0}$ are still remaining in the type I circuit as shown in Fig. $4 a$. $2 f_{0}$ and $3 f_{0}$ are suppressed to -66.9 (noise level) and $-57.3 \mathrm{dBm}$, respectively, for type II circuit in Fig. $4 b$, although some of the fundamental powers are still unsuppressed. For the proposed type III structure, all the unnecessary harmonic contents are suppressed almost to noise level, leaving the desired quadrupled harmonic components alone, as in Fig. 4c.

Measured phase noise data of the output signal of the fabricated type I, II and III quadruplers are $90.25,92.8$ and $-94.34 \mathrm{dBc} / \mathrm{Hz}$ (at $10 \mathrm{kHz}$ offset), respectively, when that of the input signal is $-101.5 \mathrm{dBc} / \mathrm{Hz}$, as shown in Fig. 5. This is an improved result compared to the theoretical phase noise degradation condition expressed by $20 \log (4)=12 \mathrm{~dB}$, with $4.74 \mathrm{~dB}$ improvement for the type III circuit. It is believed that the improved phase noise is achieved by the clear termination of the fundamental signal and unwanted harmonic signals. Fig. 6 shows frequency sweep data for conversion loss measurement. The conversion is $6.4 \mathrm{~dB}$ at $4 \mathrm{GHz}$ when the fundamental input power is $0 \mathrm{dBm}$. The conversion loss varies by $5.1-9.9 \mathrm{~dB}$ at $100 \mathrm{MHz}$ bandwidth, as shown in Fig. 6 . The variation of the loss is thought to be induced by narrowband input/output matching.

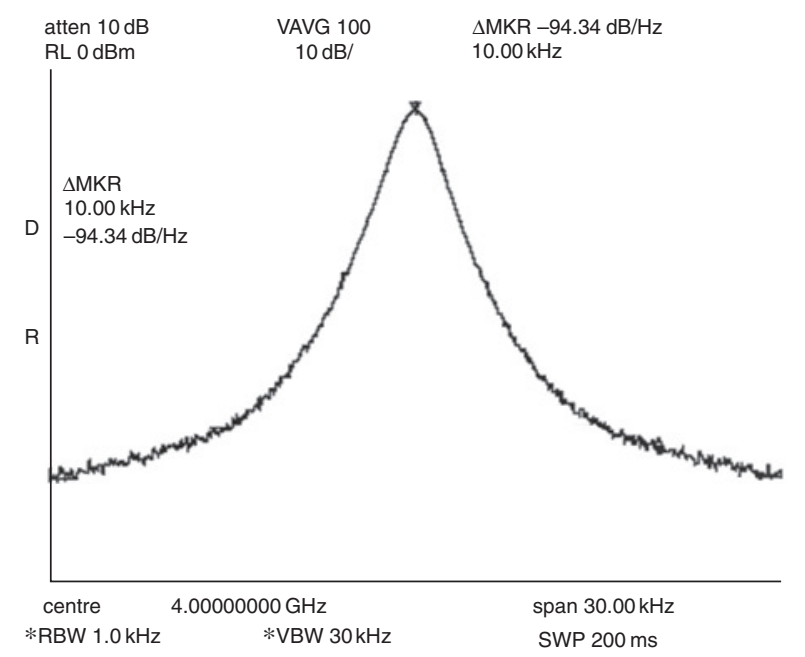

Fig. 5 Measured phase noise of quadrupled signal (input phase noise $=$ $101.5 \mathrm{dBc} / \mathrm{Hz})$

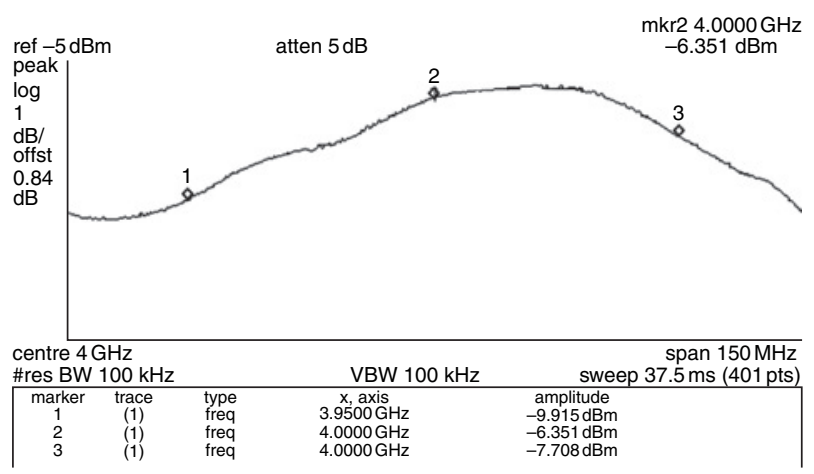

Fig. 6 Conversion loss measurement
Conclusions: A new harmonic termination method for microwave frequency quadruplers using $\lambda / 4$ CRLH open stub has been proposed and validated with experimental results. The proposed circuit is expected to be integrated in monolithic microwave integrated circuits, since the circuit components are mostly lumped elements with a bias circuitry.

(C) The Institution of Engineering and Technology 2009

20 November 2008

Electronics Letters online no: 20093331

doi: 10.1049/el:20093331

H. Choi and Y. Jeong (Information and Communication Engineering, Chonbuk National University, Jeonju, Chollabuk-do, Republic of Korea)

E-mail: streetpoet@chonbuk.ac.kr

J. Lim (Division of Information Technology Engineering, Soonchunhyang University, Asan, Republic of Korea)

\section{References}

1 Ivanov, E.N., Tobar, M.E., and Woode, R.A.: 'Ultra-low-noise microwave oscillator with advanced phase noise suppression system', IEEE Microw. Guid. Wave Lett., 1996, 6, (9), pp. 312-314

2 Tsarapkin, D.P., and Komarov, V.S.: 'Frequency stable microwave oscillator with combined frequency stabilization', Proc. Moscow Power Eng. Inst., 1973, pp. 82-87

3 Lin, I., DeVincentis, M., Caloz, C., and Itoh, T.: 'Arbitrary dual-band components using composite right/left-handed transmission lines', IEEE Trans. Microw. Theory Tech., 2004, 52, (4), pp. 1142-1149

4 Lai, A., Caloz, C., and Itoh, T.: 'Composite right/left-handed transmission line metamaterials', IEEE Microw. Mag., 2004, 5, (3), pp. $34-50$

5 Caloz, C., Sanada, A., and Itoh, T.: 'A novel composite right/left handed coupled-line directional coupler with arbitrary coupling level and broad bandwidth', IEEE Trans. Microw. Theory Tech., 2004, 52, (3), pp. $980-992$

6 Seo, S., Jeong, Y., Lim, J., Gray, B., and Kenney, J. S.: 'A novel design of frequency tripler using composite right/left handed transmission line'. IEEE Int. Microwave Symp. 2007, 2007, pp. 2185-2188

7 Cripps, S.C.: 'RF power amplifiers for wireless communication' (Artech House, 2006) 